\title{
IMPACTO FINANCIERO, TRIBUTARIO EN LA GESTIÓN ECONÓMICA SEGÚN EL SISTEMA DE DETRACCIONES
}

\author{
FINANCIAL AND TAX IMPACT IN ECONOMIC MANAGEMENT ACCORDING TO \\ THE DETRACTION SYSTEM
}

\author{
Adolfo VaienCia GuttérReZ* \\ Docente Asociado de la Facultad de Ciencias Contables \\ Universidad Nacional Mayor de San Marcos-UNMSM / Lima-Perú \\ [Recepción: Agosto 2016/ Conformidad: Setiembre 2016]
}

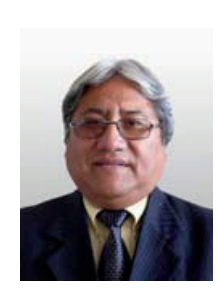

\section{RESUMEN}

El Estado ha creado regímenes de pago adelantado del IGV, a fin de ampliar la base tributaria y asegurar la recaudación y evitar la evasión. Los mecanismos aplicados, solamente han convertido a las empresas en fedatarios y recaudadores de SUNAT, se ha procedido a disminuir la liquidez de las empresas. En especial el Régimen de Detracciones, es una forma de "recaudación a ciegas” puesto que no toman en cuenta la real cuantía del impuesto que el contribuyente estaría obligado a pagar, ya que no se considera los saldos a favor o créditos tributarios de que dispone el contribuyente, quién injustamente debe distraer sus recursos financieros al pago de las detracciones complicando su flujo de caja y el valor del dinero en el tiempo. Asimismo, en el estudio se evidencia el Impacto financiero y tributario en la gestión económica de la aplicación del Régimen de Detracciones en las empresas comerciales e industriales en Lima Metropolitana, y los resultados obtenidos a través de las encuestas realizadas y el análisis de los resultados muestran el impacto negativo; La mayoría de las empresas, afrontan un excesivo endeudamiento a corto plazo, escasez de liquidez para el pago de sus obligaciones corrientes, lo cual le obliga a obtener préstamos para capital de trabajo, generando altos costos financieros por el pago de intereses.

\section{Palabras clave:}

Sistema de detracciones, sistema recaudatorio, impuesto general a las ventas, impacto financiero y económico, empresas.

\begin{abstract}
The State has created prepayment regimes of the "IGV" (GST), to broaden the tax base ensuring collection and avoiding evasion. The mechanisms applied, have only turned the companies into notaries and collectors of SUNAT, it has proceeded to diminish the liquidity of the companies. In particular, the Abatement Scheme is a form of "blind collection" since they do not take into account the actual amount of tax that the taxpayer would be obliged to pay, since the balances in favor or tax credits available to the taxpayer are not considered, who unfairly must distract their financial resources to pay the drawdowns complicating their cash flow and the value of money over time. Also, the study shows the financial and tax impact on the economic management of the application of the Abatement Regime in commercial and industrial companies in Lima Metropolitana, and the results obtained through conducted surveys and the analysis of the results show the negative impact. Most companies face excessive short-term indebtedness, a shortage of liquidity for the payment of their current obligations, which forces them to obtain loans for working capital, generating high financial costs for the payment of interests.
\end{abstract}

\section{Keywords:}

Tax abatement, collection system, general tax on sales, financial and economic impact, companies.

\footnotetext{
* Doctor en Ciencias de la Educación - UNE. Contador Público Colegiado. Email: avalenciag@unmsm.edu.pe
} 
aplicados parecieran ser medidas tolerables para los contribuyentes, en cuanto no hayan implicado un aumento de tasas, ni la creación de nuevos impuestos; sin embargo, la aplicación de estos dispositivos solamente han convertido a las empresas en fedatarios y recaudadores de SUNAT, procediéndose a disminuir la liquidez de las empresas, generando una recaudación excesiva a la real capacidad económica de las mismas. En especial el Régimen de Detracciones, es una forma de "recaudación a ciegas", puesto que no toman en cuenta la real cuantía del impuesto que el contribuyente estaría obligado a pagar, ya que no se considera los saldos a favor o créditos tributarios de los que dispone el contribuyente. Lo que se puede configurar posteriormente en el hecho de exigir adelantos a quienes no adeuden; siendo el Estado el que quedaría debiendo a los contribuyentes. Es por ello, que podemos inferir que las empresas deben injustamente distraer sus recursos financieros al pago de las detracciones, complicando su flujo de caja y el costo de oportunidad para la aplicación de la rotación de inventarios.

A esto, el empresario se convierte en una especie de propietario hipotético de "sus fondos", puesto que le pertenece "al estar en una cuenta bancaria como titular del fondo", pero no puede hacer nada con ello hasta que el Estado autorice su libre disposición, previa certificación de no adeudo. Lo cual, tampoco es automático, sino que tiene que ser solicitado como "un favor para el uso de sus fondos", siendo recién "facilitado por el Estado" previa buena voluntad de sus funcionarios. Y si hubiera incurrido en una "configuración formal" puede ser "retirado de los fondos de los contribuyentes" para ser depositados en los fondos del erario nacional. La realidad peruana en materia de informalidad y evasión tributaria, exige que se den medidas inteligentes, pero lamentablemente la real afectación recae sobre los formales; esto no sólo a los proveedores, sino también, a los adquirentes de bienes o usuarios que injustamente se ven afectados por medios que disminuyen su flujo de caja, y que decir del costo hora hombre para dar cumplimiento a la excesiva formalidad que el sistema exige, sin considerar la sustancialidad de los mismos.

\section{MARCO TEÓRICO}

En el Sistema de Detracciones el adquirente del bien o usuario del servicio sujeto al sistema, debe restar (detraer) un porcentaje del precio de venta y depositarlo en una cuenta especial, habilitada en el Banco de la Nación, a nombre del proveedor del bien o prestador del servicio. Sin embargo, el Régimen de Detracciones, genera en los contribuyentes pagos adelantados, no exigidos y muchas veces a quienes no tiene una deuda tributaria; siendo por el contrario el Estado, el que estaría debiendo a los contribuyentes. Por lo cual, podemos inferir que las empresas deben injustamente distraer sus recursos financieros al pago de detracciones, complicando su flujo de caja y el costo de oportunidad para la aplicación de la rotación de inventario. La mayoría de las empresas afrontan un excesivo endeudamiento a corto plazo, escasez de liquidez para el pago de sus obligaciones corrientes; por lo cual, requieren de préstamos generándoles altos costos financieros.

\section{Concepto doctrinal de las Detracciones}

\section{a) Definición:}

\begin{abstract}
Según Alva et al. (2013)
El vocablo "detracciones proviene del verbo detraer el cual significa "restar, sustraer, apartar o desviar" y consiste en una detracción o descuento a cargo de la persona que efectúa la compra de un bien o el usuario de un servicio que se encuentren comprendidos en el sistema, para ello se aplicará un porcentaje (\%) el cual se encuentra fijado en la norma, considerando para ello como base el Precio del Proveedor (Vendedor), para posteriormente efectuar el depósito en el Banco de la Nación, en una cuenta corriente que se encuentra a nombre del Proveedor (que puede ser el vendedor de bienes o prestador de servicios) con la finalidad que los montos depositados en dicha cuenta únicamente sean destinados al cumplimiento de los pagos de tributos del Proveedor.
\end{abstract}

Efectuar el depósito de la detracción en el Banco de la Nación en la cuenta corriente que el vendedor previamente apertura, recibiendo para ello una constancia de la cancelación respectiva, la cual sirve de sustento que la detracción sí se llevó a cabo. Posteriormente cumple con pagar la diferencia al vendedor o proveedor del servicio tomando como referencia el acuerdo comercial pactado por ellos. (p.13) 


\section{INTRODUCCIÓN}

El Sistema de Pago de Obligaciones Tributarias con el Gobierno Central - SPOT (Sistema de Detracciones) viene aplicándose en el país de manera paulatina desde el año 2002 , cuyo fin es garantizar el pago del IGV y del Impuesto a la Renta, entre otros tributos en sectores con alto grado de informalidad, sin embargo con la puesta en marcha este sistema ha distorsionado su objeto recaudatorio afectando el aspecto financiero y económico de las empresas.

Alva, M., et al (2013) Los sistemas de recaudación anticipada del IGV (detracciones, retenciones y percepciones) supone el cumplimiento de una serie de obligaciones de carácter formal y sustancial, cuya correcta ejecución ha alcanzado insospechados niveles de complejidad, formulismos, reparos y contingencias administrativas tributarias, acentuados por las recurrentes modificaciones que lejos de perfeccionar, los constituyen en sistemas cada vez más imprecisos.

En sus inicios el SPOT se aplicó únicamente a la venta de bienes y prestación de servicios gravados con el Impuesto General a las Ventas (IGV). A partir del 01 de noviembre del 2012, mediante Resolución de Superintendencia No 249-2012/SUNAT se amplía su aplicación a la venta de determinados bienes exonerados del IGV, que generan renta gravada con el Impuesto a la Renta de Tercera Categoría, los cuales se encuentran especificados en los numerales $20 y$ 21 del Anexo II de la Resolución de Superintendencia No 183-2004/SUNAT.

\section{Según, Alva et al. (2013) afirma que:}

... La generación de los fondos para el cumplimiento de las obligaciones tributarias se realizarán a través de depósitos que deberán efectuar los sujetos obligados, respecto de las operaciones sujetas al sistema en las cuentas bancarias que para tal efecto se abrirán en el Banco de la Nación. Finalmente, cabe mencionar que cuando se efectúa el depósito de las detracciones en la cuenta que el titular (proveedor) de la misma facilitó al obligado (cliente) a realizarla, no está generando el pago para efectos del cumplimiento de la prestación tributaria, ya que no se trata de una obligación a favor del estado, o no de forma directa (el dinero ha sido depositado en una cuenta corriente a nombre del proveedor), situación que confirma que no se pretende extinguir una obligación tributaria. Es decir, la detracción no califica como deuda tributaria, entendida esta última como aquella que está constituida por el tributo, las multas y los intereses tal como lo establece el primer párrafo del artículo $28 \mathrm{del}$
Código Tributario, cuyo Texto Único Ordenado fue aprobado por el Decreto Supremo No. 133-2013-EF y modificatorias, por lo que no es aplicable las normas del Código Tributario. Incluso para recurrir las decisiones administrativas derivadas del SPOT, se debe utilizar los recursos administrativos previstos en la Ley del Procedimiento Administrativo General, Ley $N^{\circ} 27444$, como el de Reconsideración o Apelación. (p. 11)

Ahora bien, la puesta en marcha del sistema de Detracciones como lucha contra la evasión tributaria y la informalidad, se ve de cierto modo distorsionado en su objetivo recaudatorio, de manera tal, que desnaturaliza su fundamento base, al no tener en cuenta los principios constitucionales al que se sujeta toda ley o norma tributaria al ser formulada. En nuestra constitución podemos encontrar fundamentalmente los principios explícitos e implícitos en el artículo $74^{\circ}$ de la Constitución Política del Perú del año 1993. Por otro lado, en cuanto a la facultad sancionadora de la Administración Tributaria, en el caso de las pequeñas empresas, es inadecuado; puesto que, por faltas no significantes se les sanciona con multas y cierre del local, actitud que no realiza con la gran empresa. Y por el hecho de que la Administración Tributaria goce de facultades discrecionales, no implica, de modo alguno, que estas puedan ejercerse de modo irrestricto, arbitrario o ilimitado. Sin embargo, el contribuyente que cumpla con aportar, asimila su contribución como un costo, sin tener manera alguna de beneficiarse directamente. Así, el costo individual podría ser muchísimo más grande que el beneficio común, donde en realidad este aporte del contribuyente debe tender a una relación que se base en la razonabilidad, entre el costo individual y el beneficio colectivo, porque eso permitirá que la cultura tributaria crezca y el propio país sea gobernable.

En realidad la forma de generar ingresos para el Estado, mediante el pago adelantado del IGV, afecta financiera y económicamente a las empresas. La buena intención se ve perjudicada, al ocasionar problemas al desarrollo de las empresas, cuando éstas realizan ventas a crédito, por efecto de pagar dicho cobro por adelantado, sin que hayan recibido el dinero aludido en la norma tributaria. En realidad el cobro por adelantado del IGV a las empresas que realizan ventas al crédito, sí perjudican financiera y económicamente al desarrollo empresarial. Los mecanismos 


\section{b) Naturaleza jurídica de las Detracciones}

\section{Gestión del error}

\author{
Según Alva et al. (2013)
}

La obligación por parte del adquirente o usuario de depositar una parte de la retribución previamente acordada con la persona que califica como proveedor de bienes o servicios, en una cuenta abierta a nombre de este último en el Banco de la Nación, como se ha dicho, no califica como un tributo. Una de las características que define a nivel jurídico al tributo es el de consistir en una obligación de entregar dinero al Estado, quien en ejercicio de su facultades coercitivas exige el cumplimiento de la prestación tributaria a cargo del contribuyente a favor del acreedor tributario que es el Estado, situación distinta se presenta en el caso de la detracción, toda vez que no es el Estado el acreedor sino el proveedor de los bienes o servicios que calificará como acreedor del porcentaje a depositar en su cuenta producto de la detracción. Así, la detracción al no calificar como tributo tiene como consecuencia que no extingue deuda tributaria, no se le pueden aplicar intereses moratorios si se incumplen con el pago de la misma (pero si a la multa), no puede ser materia de cobranza coactiva por parte de la Administración. De este modo el adquirente no califica como contribuyente ni tampoco como responsable”. (p.14)

\section{c) Aspectos conceptuales de los Estados Financieros}

Olano, H (1974) "Los estados financieros "conforman los medios de comunicación que las empresas utilizan para exponer la situación de los recursos económicos y financieros a base de los registros contables, juicios y estimaciones que son necesarios para su preparación" (p.15) Zeballos, (2007).

En base a esta conceptualización podemos decir entonces que los Estados Financieros, muestran la situación económica y financiera o el resultado en la gestión de la empresa durante un periodo de tiempo determinado, expresando de esta forma en cuadros sinópticos, los datos extractados de los libros y registros contables. Los Estados Financieros condensan la información que ofrecen las cuentas contables y las clasifican de acuerdo con los principios de contabilidad generalmente aceptados para establecer: - La situación y el valor real del negocio; y - El resultado de las operaciones con una fecha determinada. (p.343).

\section{d) Consideraciones de los ratios financieros}

\section{Liquidez}

La liquidez es la capacidad de la empresa de hacer frente a sus obligaciones básicamente en un corto plazo. La liquidez se define como la capacidad que tiene una empresa para obtener dinero en efectivo. Es la proximidad de un activo a su conversión en dinero.

La liquidez es la posesión de la empresa de efectivo necesario, en el momento oportuno que le permita hacer el pago de los compromisos anteriormente contraídos. Representa la cualidad de los activos para ser convertido en dinero efectivo de forma inmediata sin pérdida significativa de su valor. De tal manera, que cuanto más fácil es convertir un activo en dinero más líquido se dice que es. Para medir la liquidez de una empresa se utiliza el ratio o razón de liquidez. La cual mide la capacidad de la empresa para hacer frente a sus obligaciones a corto plazo. Del análisis de estas razones se puede conocer la solvencia de efectivo de la empresa y su capacidad de permanecer solvente en caso de acontecimientos adversos.

\section{e) Finalidad de los estados financieros}

Los estados financieros constituyen una representación estructurada de la situación financiera y del rendimiento financiero de la entidad. El objetivo de los estados financieros, con propósitos de información general, es suministrar información acerca de la situación del rendimiento financiero y de los flujos de efectivo de la entidad, que sea útil a una amplia variedad de usuarios, a la hora de tomar sus decisiones económicas. Los estados financieros también muestran los resultados de la gestión realizada por los administradores, con los recursos que se les han confiado. Se puede colegir entonces, a la luz de los datos obtenidos en la investigación, mediante las encuestas desplegadas, que el impacto financiero tributario en la gestión económica de la aplicación del régimen de detracciones en las empresas comerciales e industriales en Lima Metropolitana es negativo. 


\section{RESULTADOS}

A continuación se presentan los resultados del trabajo de campo en la investigación.

En la figura No 01 , se observa que el $60 \%$ de los encuestados opinan que sí sabe que los fondos de- positados en la cuenta de detracción cumplen su fin. Pero el $40 \%$ no usan los depósitos para ello; lo cual, le quita un valioso costo de oportunidad pudiendo usarlo más productivamente en los pagos de tributos.

\section{Figura No 01}

Fondos depositados en cuenta de Detracciones

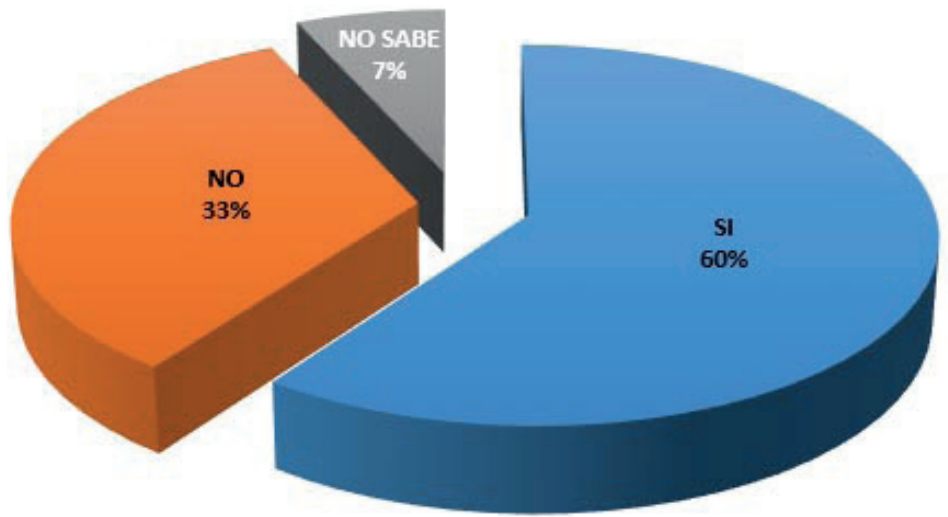

Fuente: Elaboración propia.

En la Figura No 02, se aprecia que solo el 13\% cumplen con pagar en el tiempo oportuno sus tributos. En su mayoría acumulan un pasivo tributario que a la postre le puede generar un nivel de insolvencia. El 54\% no cumple y el $33 \%$ prácticamente desconoce el tema.

\section{Figura No 02:}

Cumplimiento de los proveedores sujetos al SPOT

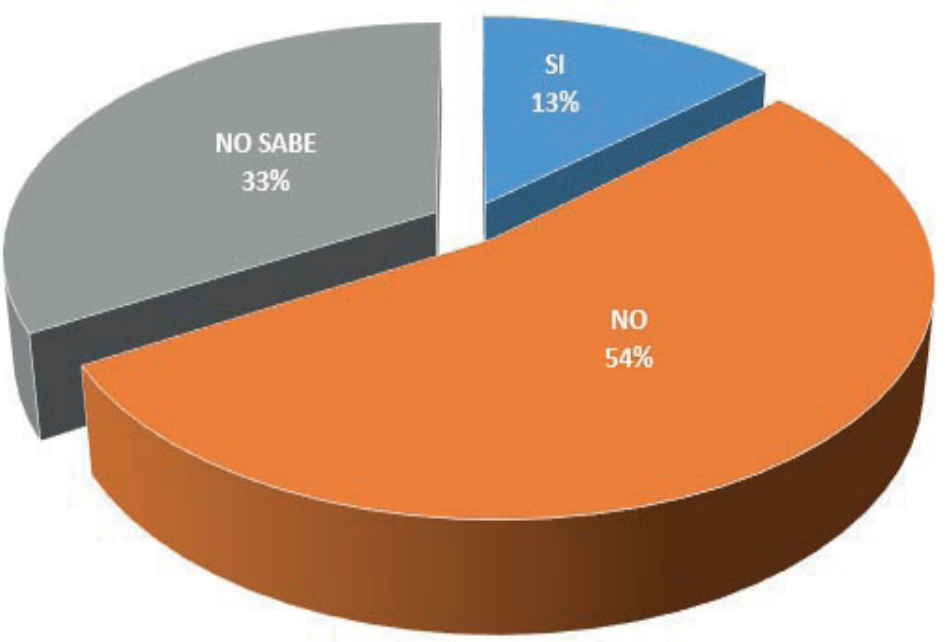

Fuente: Elaboración propia. 
Se sabe que el dinero tiene un valor en el tiempo, para el caso el $100 \%$ de encuestados, que sienten que da de ganar al banco, pues es un dinero ocioso que no remunera. Perdiéndose un valioso costo de oportunidad. Tal como se aprecia en la figura $\mathrm{N}^{\circ}$ 03, que muestra el promedio de inmovilización detracciones en meses.

\section{Figura No 03}

Promedio de inmovilización de las detracciones

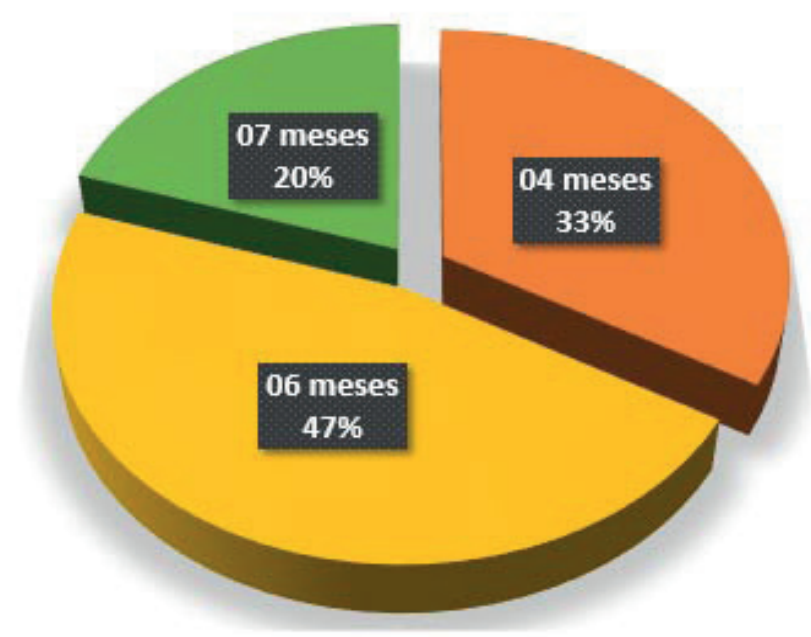

Fuente: Elaboración propia.

En la Figura No 04, se observa que el $80 \%$ sabe, que al inmovilizar fondos, se impacta en la gestión financiera, pues la empresa pierde liquidez; perdiendo

la oportunidad de usar fondos en otras alternativas. Sin embargo, se hace la cultura financiera del pobre.

\section{Figura No 04}

Impacto Financiero por inmovilización

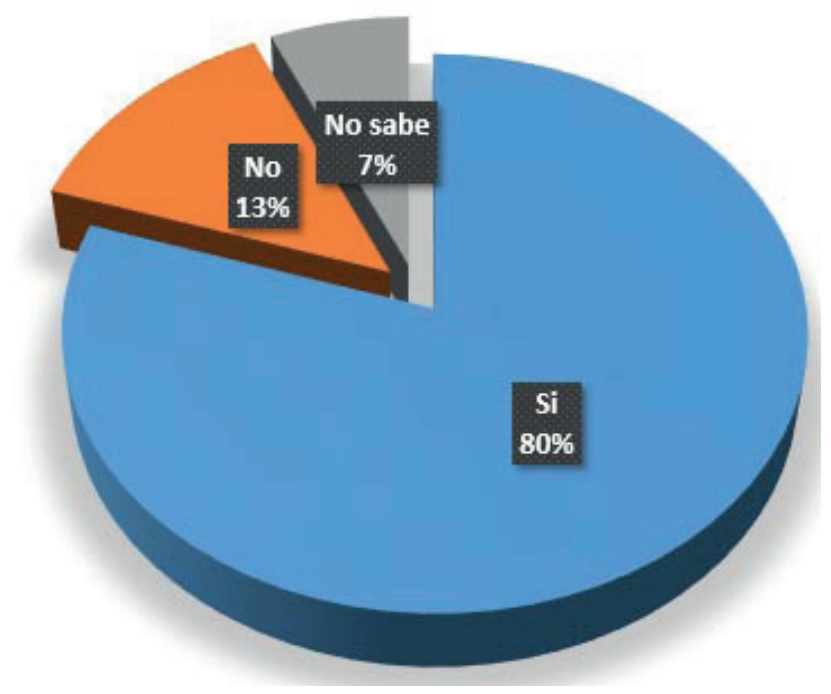

Fuente: Elaboración propia. 
Si hablamos de generar gastos financieros, el $87 \%$ es consciente que el inmovilizado, genera costos financieros, teniendo que recurrir a otras fuentes de financiamiento para cumplir sus necesidades de capital de trabajo, asi como denota la Figura No 05 .

\section{Figura No 05}

El depósito de detracciones genera costos financieros

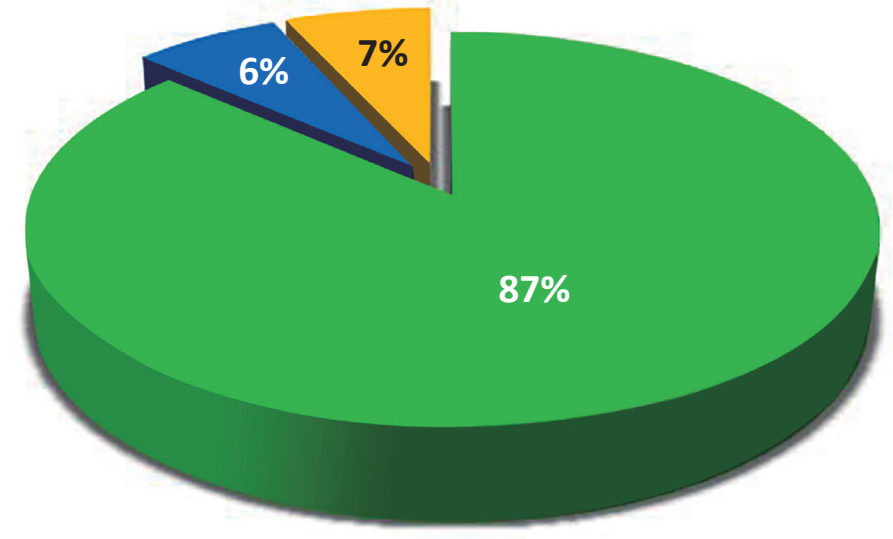

$$
\square \text { Si No } \square \text { No sabe }
$$

Fuente: Elaboración propia.

En tanto, en la Figura No 06, el 93\% opina afirmativamente, es decir ven al sistema de detracciones como una herramienta que los asfixia más, viendo a la formalidad con más desventajas que ventajas.

Figura No 06

Tasas altas de las detracciones VS. Los impuestos como MYPE

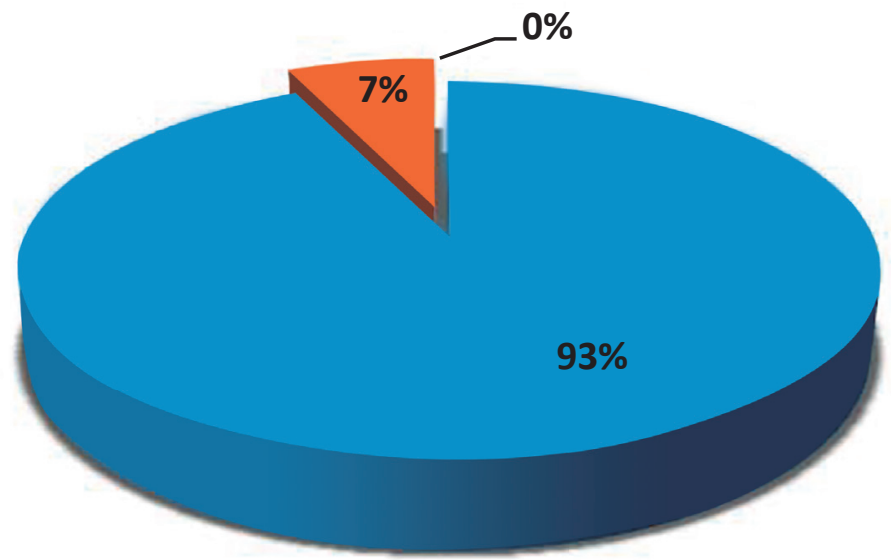

$$
\text { Si No No sabe }
$$

Fuente: Elaboración propia. 
La Figura No 07 muestra, que un $40 \%$ desconoce que al no hacer el pago oportuno de las detracciones, pierde la oportunidad de hacerse acreedor de un cré- dito fiscal. Lo cual le da menos grados de libertad a la empresa, en su manejo de capital de trabajo.

\section{Figura No 07}

El no pago del SPOT impide el uso del Crédito Fiscal

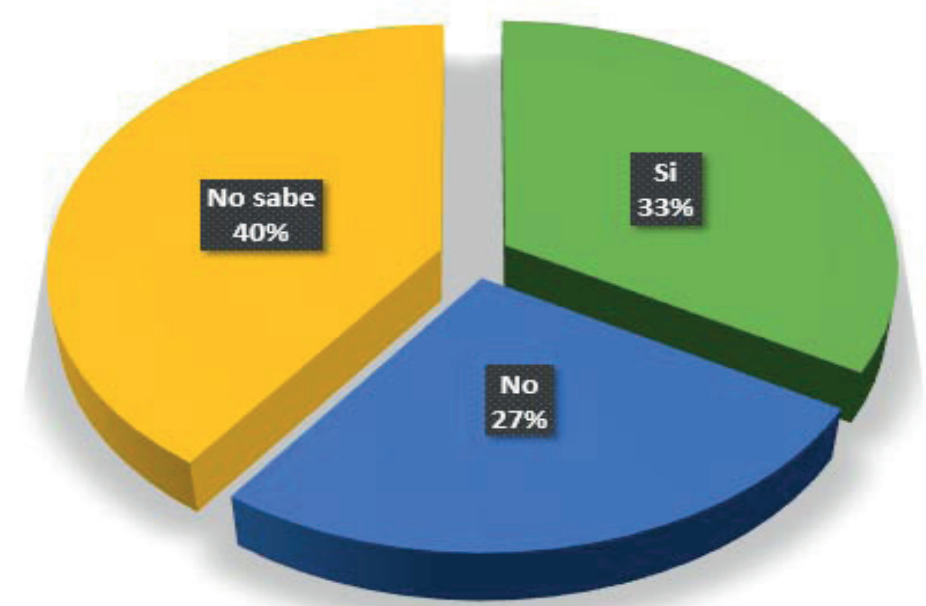

Fuente: Elaboración propia.

Por último, en la Figura No 08, observamos que el $67 \%$ opina que no puede disponerse de las detracciones para pagar deudas tributarias; mientras que el
$13 \%$ opina que la entidad mencionada, sí puede disponer de la cuenta de Detracciones.

\section{Figura No 08:}

SUNAT puede disponer de la cuenta de Detracciones para cobro de las deudas tributarias

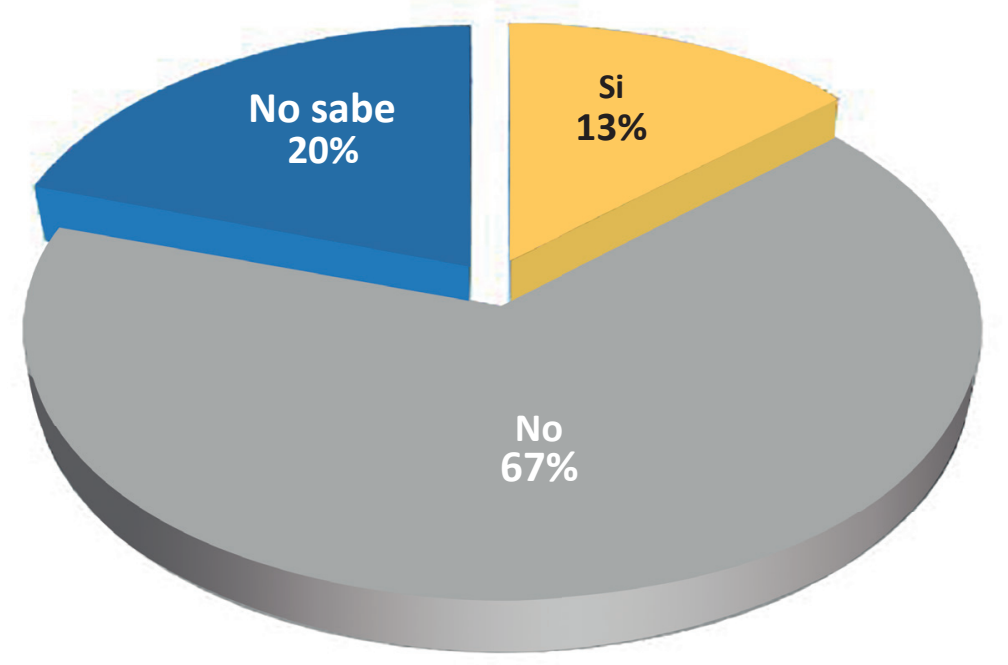

Fuente: Elaboración propia. 


\section{CONCLUSIONES}

1. La aplicación del sistema de cobro por adelantado de las Detracciones, influye negativamente en el aspecto económico financiero de los contribuyentes.

2. El Sistema de detracciones constituye un sistema de ahorro forzoso y por ende, colisiona con el derecho fundamental a la propiedad, pues, precisamente impide el ejercicio de uso y disfrute del dinero, elementos que constituyen componentes esenciales de dicho derecho.

3. Asimismo, las empresas se ven injustamente obligadas a detraer parte de sus recursos líquidos al pago adelantado del IGV, afectando la liquidez de la empresa, debido a que no reciben el monto total planeado por la venta de sus bienes o la prestación de sus servicios; contando con menores posibilidades de reinvertir su capital de trabajo.Además, se ve obligado a recurrir a entidades financieras para obtener préstamos o sobregiros y así cubrir sus obligaciones a corto plazo.

\section{REFERENCIAS BIBLIOGRÁFICA}

1. ALVA, M., GARCÍA,J., GUTIÉRREZ, L., PEÑA, J., BERNAL, J., MORILLO, M., FLORES, J. (2013) Manual de Detracciones, Retenciones y Percepciones. Pacífico Editores, Lima, Perú.

2. OLANO, H (1974) “Los estados financieros" s/f Lima, Perú

3. ARANCIBIA, M (2005) Manual del Impuesto General a las Ventas e Impuesto Selectivo al Consumo, Pacífico Editores, Lima, Perú

4. LAWRENCE J. GITMAN (2003) Principios de Administración Financiera, Pearson, México. http://repositorio.upao.edu.pe/bitstream/upaorep/203/1/CASTRO_PA\%C3\%9AL_FACTOR\%C3\%8DA COMERCIAL TRANSPORTE.pdf (visitado el 3/04/2015)

5. http://orientacion.sunat.gob.pe/index.php/ empresas-menu/regimen-de-detracciones-del-igv-empresas/como-funcionan-las-detracciones (visitado el 03/04/2016) 